\title{
Dental Practice Guidelines in the Precariousness of COVID-19: A Review
}

\section{Gargi Nimbulkar ${ }^{1}$, Niharika Dubey², Shipa Mandwar', Samiksha Dharmapuria², Amit Reche', Kumar Gaurav Chhabra ${ }^{3}$}

\begin{abstract}
'Assistant Professor, Department of Public Health Dentistry, Sharad Pawar Dental College and Hospital, Datta Meghe lnstitute of Medical Sciences (Deemed to be University), Sawangi (Meghe), Wardha, Maharashtra-442001, India; ${ }^{2}$ Assistant Resident, Sharad Pawar Dental College and Hospital, Datta Meghe Institute of Medical Sciences (Deemed to be University), Sawangi (Meghe), Maharashtra-442001, India; ${ }^{3}$ Associate Professor and Head, Department of Public Health Dentistry, Sharad Pawar Dental College and Hospital, Datta Meghe Institute of Medical Sciences (Deemed to be University), Sawangi (Meghe), Wardha, Maharashtra-442001, India.
\end{abstract}

\section{ABSTRACT}

The rapid spread of novel corona virus declared as a pandemic by the World Health Organisation has created a sense of panic across the globe, nor sparing the Dentistry in the process. The mode of transmission of the virus is through infected secretions such as saliva and respiratory secretions or their respiratory droplets, which are expelled when an infected person coughs, sneezes, talks or sings, has created the need to control the contact spread of the infectious virus between humans. This demanded a responsible emergence of medical as well as Dental care to handle the situation of the pandemic with utmost care. The need for proper guidelines from time to time has been a key to the emerging safe dental practices amongst dental professionals. Focusing not only on the emergencies but also on the routine procedures is a major challenge at the present. The best delivery of dental treatment can only be given when proper protocols are being followed. A crucial aspect being the protection of the spread of the disease from an asymptomatic patient should be considered. This review has been articulated considering the dire need of the hour to protect the dental professionals, the auxiliaries as well as the patient from the infectious spread of the disease. It encourages them to know the various methods of the protective aspects of dentistry amidst this pandemic and its importance in clinical practice. Also, additional aspects of precautions while screening the patient, while performing various procedures and the disinfection of the operatory has been discussed.

Key Words: Aerosol transmission, COVID-19, Dentistry, Guidelines, Prevention

\section{INTRODUCTION}

The novel coronavirus disease orCOVID-19, a disease manifesting as a series of idiopathic pneumonia cases has led to $>6$ million confirmed cases and 383,262 deaths in 216 countries/territories around the world as of June 03, 2020. ${ }^{1,2}$ With a lot of research going around the disease, the currently known transmission route is via the contact and through droplets, however, the airborne spread is although uncertain but possible. The transmission through aerosol and fomite is also reasonable because the virus can remain viable and infectious in aerosols for as long as 3 hours and for days on surfaces. ${ }^{3}$ A number of dental procedures produce contaminated aerosols and droplets that have the potential for infection transmission to the dental healthcare provider, patients and staff along with their families in the dental office. ${ }^{1}$ Also certain groups need cautious management such as geriatric patients, diabetics, hypertensive and anaemic patients who are at risk of the nosocomial spread than patients with no underlying conditions. ${ }^{4}$

In the wake of this pandemic, government dental organizations and private practitioners have been asked to close their practices $^{5}$ to prevent the fire of COVID-19 from spreading. On the contrary, patients presenting with extensive dental pain not alleviated by painkillers or even more severe potentially fatal dental emergencies require immediate attention from the dental health care provider and thus exigent dental procedures are essential to be catered to the people even during the pandemic. ${ }^{6}$ Hence, organizations and practitioners have been permitted to treat such patients while maintaining all the safety protocols to prevent COVID-19 from spreading. 5,7

\section{Corresponding Author:}

Dr. Kumar Gaurav Chhabra, Associate Professor and Head, Department of Public Health Dentistry, Sharad Pawar Dental College and Hospital, DattaMeghe Institute of Medical Sciences (Deemed to be University), Sawangi (Meghe), Wardha, Maharashtra, India- 442001 Mobile: 7976840339; Email: rajsushil.chhabra@gmail.com

ISSN: 2231-2196 (Print)

Received: 11.06 .2020
ISSN: 0975-5241 (Online)

Revised: 09.08.2020
Accepted: 23.09 .2020
Published: 06.10 .2020 
The release of airborne material in certain procedures of dentistry is quite obvious. An aerosol is a combination of particulate matter and fluid often forming a cloud over the working area. This cloud can be observed during the use of two-way syringes, preparation of tooth by rotary instrument, ultrasonic scaling and air abrasions. The individuals coming in contact with those particles are dentist, dental team and patients. With the advent of novel Coronavirus, concern regarding the potential spread of infections from this aerosol splatter has risen from the death bed. ${ }^{8}$

Hence, it becomes important to throw light on the functioning of dental healthcare systems in the times of COVID-19 pandemic, highlighting the judicious measures to be adopted to provide the best treatment possible keeping in mind the safety of the dental staff as well as the patients.

\section{DECISION MAKING FOR PERFORMING TREATMENT}

While shutting down dental setups reduces the transmission, thereby helping in reducing the number of people affected with the virus, it will increase the number of people suffering from dental conditions requiring immediate attention and such patients can increase the burden on the emergency departments in hospitals. ${ }^{9}$ Correspondingly, the American Dental Association on 16 March 2020 issued guidelines which stated that dentists across the nation should put off all elective dental procedures and treat only cases that required urgent attention, initially for 3 weeks and later extended the same to $30^{\text {th }}$ April. ${ }^{5}$ The Dental Council of India in its circular released about the mandatory precautionary measures for the dentists to be applied during

COVID-19, dated 17 March 2020, clearly stated that clinical procedures involving the generation of aerosols should be avoided and only emergency procedures are to be carried out. ${ }^{7}$ Keeping in mind the current scenario of India and abroad, dentists have still not been permitted to carry out routine dental procedures and in case of emergency, the patient should be handled with utmost care with mandatory prevention protocols laid down by various institutions. ${ }^{5,7}$

\section{EMERGENCY DENTAL CONDITIONS}

Excruciating dental pain which is not alleviated by analgesics or even more severe potentially fatal dental emergencies like torrential bleeding from the oral tissues, head and neck, facial space infections or trauma involving the face possibly compromising patient's airway require immediate attention from the dental health care provider. ${ }^{6}$ Having said that and knowing the pathogenicity and the immense infectiousness of the disease, it becomes critical for the dental institutions and offices to respond to the situation. Dental emergencies specified by American Dental Association include uncon- trolled bleeding, cellulitis, trauma involving the facial skeleton, possibly restricting the airway, extensive dental pain due to inflammation of the pulp, pericoronitis or pain from the $3^{\text {rd }}$ molar and trauma to the tooth including avulsion/ luxation. ${ }^{5}$ Dental emergencies can come at any time in a dental setup. The dentist as well as the dental nurses should be prompt in their responses to the emergencies. It was found in institutional research that the response of the female dental nurses is more significant and efficient compared to males in primary emergencies. ${ }^{10}$

\section{STEPS TO PROCEED FOR EMERGENCY CARE}

\section{A) PATIENT SCREENING}

While managing a dental emergency patient in an ongoing pandemic, certain measures become mandatory to be adopted based on current knowledge of the extensive pathogenicity and spread of SARS-CoV- 2. A set of protocols as mentioned below should be followed in every dental setup:

1. Telescreening and Teleconsultation are advisable for early recognition, prevention of transmission and to ensure triage.

2. Carefully screen every patient including the history of contact, travel history and symptoms and mandatory thermal screening (infrared thermometer). ${ }^{11}$

3. Consider each patient to be a potential COVID-19 carrier.

4. Consider patients who have recovered recently to be potential carriers for 30 days and recovery confirmed by a lab test.

5. Identify the urgency with the prime focus on minimally invasive procedures.

6. Categorise dental procedures according to how urgently the treatment is required along with associated risks and benefits.

Use necessary precautions against contact and procedures generating aerosol. ${ }^{9}$

When a patient arrives, the patient should fill up the following -

1. Medical history form.

This includes the current state of consciousness, general examination (Temperature, pulse, respiratory rate, Blood pressure) history about any genetic illness, allergy to a certain drug, previous history of any surgery or any known illness.

2. COVID-19 screening form. ${ }^{12,13}$

It generally includes the basic demographic details especially address along with travel history and also include questions regarding symptoms like cold, fever and sore throat.

3. Emergency assessment questionnaire. ${ }^{14}$ 
This includes the current status of bleeding, pain and respiratory difficulties or any other severely discomforting symptom which gives the dentist an idea about the urgency of the situation and whether a prompt treatment is required or not.

Any suspected patient is asked to engage in self-quarantine along with consultation from the physician with regards to his/her symptoms. Self-quarantine protocols should be strictly followed as per the guide

\section{PREVENTIVE MEASURES THAT CAN REDUCE THE POTENTIAL SPREAD}

To reduce the possibility of spread in dental healthcare facilities, the following algorithm for risk assessment (Table-1) was given by the United States Department of Labour. Thus, administrative measures like staff reduction protocols following adequate patient-staff ratio and proper training of the staff carried out, minimises risk for other dentists, dental auxiliaries and patients should have opted.

Table 1: Algorithm for risk assessment ${ }^{15}$

\begin{tabular}{|c|c|c|c|}
\hline Lower (caution) & Medium & High & Very High \\
\hline $\begin{array}{l}\text { Administrative duties } \\
\text { in non-public areas of } \\
\text { dentistry facilities }\end{array}$ & $\begin{array}{l}\text { Provision of non-aerosol-gen- } \\
\text { erating urgent or emergency } \\
\text { dental care to the general } \\
\text { public who are not known or } \\
\text { suspected COVID-19 patients. } \\
\text { Provision of services in busy } \\
\text { staff work areas within a den- } \\
\text { tistry facility. }\end{array}$ & $\begin{array}{l}\text { Entry in a known or suspected COV- } \\
\text { ID-19 patient's room or care area. } \\
\text { * Provision of non-aerosol-generating } \\
\text { dental care service to a known or sus- } \\
\text { pected COVID-19 patient. } \\
\text { Provision of aerosol-generating dental } \\
\text { care service to the general public who } \\
\text { are not known or suspected COVID-19 } \\
\text { patients. }\end{array}$ & $\begin{array}{l}\text { Provision of aerosol-gen- } \\
\text { erating dental care service } \\
\text { to known or suspected } \\
\text { COVID-19 patients. } \\
\text { Collection of or handling } \\
\text { specimens from known } \\
\text { or suspected COVID-19 } \\
\text { patients. }\end{array}$ \\
\hline
\end{tabular}

\section{PREVENTIVE MEASURES FOR DENTISTS AND AUXILIARIES}

When the patient comes for consultation.

1) Patient is asked to wash his/her hands with soap thoroughly and a mask is provided in the waiting area.

2) Infrared thermal screening of every patient.

3) Isolated consultation room which is not directly in contact with the waiting area. The waiting chairs should follow a social distance of $1 \mathrm{~m}$.

4) On arrival a patient is provided mask, head cap, shoe cover and gloves to wear.

5) During a consult the dentist and auxiliary should compulsorily wear a head cap, eyewear, face-shield and a mask that is TRIPLE LAYERED or a 3 ply mask along with gloves. The Personal Protective Equipment (PPE) should be up to 60-75 gram per square metres (gsm). A gown along with shoe cover should be used.

\section{During operatory procedures}

It is a routinely observed scenario in dental clinics that the patients lack knowledge regarding the cross-infection spread in a clinic and are often ignorant about it. So it becomes the duty of the clinician to protect them as well as themselves from cross infections of the operatory. ${ }^{16}$

An operatory procedure is either aerosol producing or a nonaerosol producing.

Any procedure should begin with asking the patient to rinse his mouth with 1\% Hydrogen Peroxide or $0.2 \%$ Povidone
Iodine one minute before the procedure and should also be scrubbed extra-orally with an antiseptic wipe..$^{6,17,18}$

\section{PROTECTION IN A NON-AEROSOL PRODUCING PROCEDURE}

Personal Protective Equipment includes eyewear, faceshield, mask, gloves and coverall/gowns. These should be up to $80-100 \mathrm{gsm}$. It shields the skin and mucous membranes from subjection to contagious substances. The possibility of splash or splatter necessitates the use of a shield. Recommended sequences to be followed for donning and doffing PPE has been given by CDC. ${ }^{19}$

1) Masks, Protective Eyewear, Face Shields

- For non-aerosol protection according to American Society of Testing and Materials, ASTM TYPE 1 maskswith fluid resistance of $80 \mathrm{mmHg}$ and 95 $\%$ bacterial filtration can be used along with faceshield or eyewear.

- These are specific Respirator kinds of masks.

2) Changing of masks can be done after every patient or during the same patient if it is wet.

3) Protective Clothing

- Lab coats/gowns covering skin and personal attire that might get stained with infectious substances including saliva and blood. Also, shoe-covers to be used along with the coveralls.

- In case of visible stains, change.

- All the barriers are taken off before leaving the work area. 
4) Gloves

- Prevent contamination of dental healthcare provider's (DHCP) hands while they come in contact with mucous membranes or any possible contagious substance, including saliva and blood.

- Minimize the possibility of spread of micro-organisms from the hands of DHCP to patients while performing surgical or any other procedure.

- This does not banish the requirement for washing the hands.

Hand hygiene is promptly performed after getting rid of the gloves post patient procedure..$^{20,21}$

\section{PROTECTION DURING AN AEROSOL PRODUCING PROCEDURES}

Table 2: Protection to be used for aerosol producing procedures

Protection Description

Personal Protective Protection from the hazardous exposure to the aerosols (particle size of the diameter of 50 microns and Equipment less) using the thickness of up to 100 gsm along with a single-use disposable cover gown.

Masks, Protective Eyewear, Face Shields

According to the American Society for Testing and Materials(ATSM), for aerosol producing procedures use of a fitted N-95 respirator (that filters particles up to $0.3 \mu \mathrm{m}$ ). These are ASTM TYPE 2 or 3 with fluid resistance of $120 \mathrm{mmHg}$ and more, with $98 \%$ bacterial filtration. Every mask should be changed after each procedure. Protective eyewear with flexible frames to give a perfect eye fit is to be used. Face-shields are made of plastic that gives clear visibility and prevents fogging. It has an adjustable headband for a close fit to cover the entire face.

Protective Clothing Put on long-sleeved lab coats or gowns covering skin and personal attire that might get stained with infectious substances including saliva and blood but these might not suffice. Use of disposable coverall gowns for 36odegree protection that protects even back and a mid-calf length. These are also available with thumb and finger slots. All the coveralls should be South India Textile Research Association [SITRA] approved. Standard specifications are ISO 16603 class 3 exposure pressure or equivalent. Shoe covers can be used along with overalls for complete protection. Head caps are mandatory for the dentist as well as the auxiliary.

Gloves

Use of nitrile gloves that extend up to mid-forearm [upto $280 \mathrm{~mm}$ ] can be done. Other types may include regular latex variety. Sizes are 6.5and 7. Quality equivalent: EU standard directive 93/42/EECClassI,EN455, EU standard directive 89/686/EEC Category Ill, EN 374, ANSI/SEA 105-20110r ASTM D6319-10

\section{Table 3: Dental operatory precautionary measures- Recommendations for disinfection}

\section{Surface Disinfection Methods}

1. Avoiding the use of air-water syringe and promoting the use of high volume suction tip and the use of rubber-dam to reduce salivary contamination has been recommended. ${ }^{21}$

2. Regular cleaning and procedures for disinfection of surfaces by applying an Environmental Protection Agency registered ${ }^{23}$, hospital-grade disinfectant to surfaces touched often like door handles, nozzles of taps, switches, dental chair and parts of dental chair etc. are appropriate for COVID-19 prevention in healthcare setups, plus those areas where procedures generating aerosols are carried out. ${ }^{21}$

3. Disinfection of surfaces with $0.1 \%$ sodium hypochlorite, $70 \%$ ethanol, $1 \%$ povidoneiodine or $0.5 \%$ hydrogen peroxide for one to five minutes can effectively inactivate the virus. $^{24,25}$

4. Use of UV light disinfection for polyvinyl siloxane impressions is better than using $2 \%$ Glutaraldehyde or $1 \%$ sodium hypochlorite to preserve their dimensional stability. ${ }^{26}$ Floor mopping with $0.1 \%$ sodium hypochlorite. ${ }^{21}$

\section{Air Disinfection Methods}

1. For aerosol contamination use of Foggers, High-efficiency particulate air purifier(HEPA), Ultraviolet(UV) germicidal irradiation or negative ion generator to lower the aerosol contamination has been suggested. ${ }^{21}$

2. Methods employed for fumigation, the electric boiler fumigation method is most commonly performed which includes dilution of $500 \mathrm{ml}$ of formaldehyde in 10ooml of water in an electric boiler for every $1000 \mathrm{ft}^{3}$. The other method is the potassium permanganate method that involves the addition of $450 \mathrm{gm}$ of $\mathrm{KMNO}_{4}$ to $500 \mathrm{oml}$ of formaldehyde for every $1000 \mathrm{ft}^{3}$ to generate formaldehyde fumes as a result of auto boiling. ${ }^{27}$

3. Fogging, a procedure employed for air disinfection uses a solution of $11 \%$ hydrogen peroxide and $0.01 \%$ of silver nitrate for the preparation of $20 \%$ solution. 45 minutes is the fogging time which is followed by a contact time of 1 hour. ${ }^{21}$

\section{Miscellaneous}

1. Reusable equipment used for patient treatment are to be disinfected as suggested by the manufacturer in conjugation with facility policies. ${ }^{22}$ Around 30 seconds of flushing the water pipelines of handpieces with disinfectant solutions and handpiece cleaning by a handpiece cleaning solution followed by autoclaving.

2. Management of clinical waste should be as per regular procedures. $^{21}$

3. As far as radiography is considered, extra-oral radiography viz panoramic and cone-beam computed tomography are preferred over intra-oral radiographic imaging to circumvent cough or gag reflex. Providing double barriers to the sensors avoids cross-contamination in case of intraoral radiography. ${ }^{14}$ 4. Safe handling of samples. ${ }^{7,18}$ 


\section{DISCUSSION}

COVID-19 has emerged as a global pandemic across the world. It had rendered the world standstill, including dentistry. With lots of guidelines, it becomes confusing as to what is to be exactly done. All the leading agencies and organisation in dental health care sectors have put efforts, now we have guidelines about what is to be done is given circumstances. Dentistry has entered the era of safer practice policies given a pandemic. Considering emergency therapy as a priority, use of protective equipment became crucial for dentists as well as patients. Slowly, it has now been possible to deliver elective procedures to the patients along with the help of proper safety protocols. As the name suggests these are the guidelines and the final call is dependent on the dentist's judgement. A role of the dentist in such a pandemic is not only to treat their patients but also to prevent the transmission of the disease. Hence, the safety of the patient as well as the clinician is equally important for preventing further incidences of this disease. This review summarises the important points in the whole manuscript which stresses on the importance of Screening and triage at the point of entry of patients into the health care system, only those patients requiring emergency treatment should be entertained. With the full personal protective types of equipment on, the dental health care provider should take thorough medical history which includes COVID 19. The patient is necessarily asked to undergo basic hygiene procedures to wash his/her hands with soap thoroughly followed by Infrared thermal screening and isolated consultation room with waiting chairs placed at a distance of $1 \mathrm{~m}$. An operatory procedure which is either aerosol producing or a non-aerosol producing should begin rinsing the patient's mouth with $1 \%$ Hydrogen Peroxide or $0.2 \%$ Povidone Iodine one minute before the procedure and should also be scrubbed extra-orally with an antiseptic wipe. After the procedure, the biomedical waste generated should be disposed of safely. The phenomenon of Donning and Doffing of the PPE should be followed thoroughly and meticulously. As the various states in India have witnessed increased in cases ${ }^{28,29}$, it is the duty of the health care professional to safeguard the health of himself, the patient's and ultimately of the nation by following the protocols as per described by the higher authorities.

\section{CONCLUSION}

After assessing and understanding the current scenario of the on-going pandemic, dentists can play a major role in preventing the transmission of COVID-19. Abiding by the prevention protocols and judiciously carrying out the disinfection procedures is the key to pass through the large stubborn door of this pandemic. Not only emergency conditions have to be compulsorily attended by the dentists across India but also moving towards newer methods of practising dentistry like Teleconsultation and online consultation has to be incorporated. The private practitioners are the worst hit from this pandemic as the guidelines of the country regarding pandemic strictly prohibits them from practising and currently are in shut downstate. Therefore a dentist will have to balance the act of prescribed guidelines accurately and prompt provision of emergency services to the patient.

Acknowledgement: Authors acknowledge the immense help received from the scholars whose articles are cited and included in references of this manuscript. The authors are also grateful to authors / editors / publishers of all those articles, journals and books from where the literature for this article has been reviewed and discussed.

\section{Conflict of Interest: Nil}

\section{Source of Funding: Nil}

\section{REFERENCES}

1. Guo H, Zhou Y, Liu X, Tan J. The impact of the COVID-19 epidemic on the utilization of emergency dental services [published online ahead of print, 2020 Mar 16]. J Dent Sci. 2020;10.1016/j. jds.2020.02.002. DOI:10.1016/j.jds.2020.02.002

2. World Health Organization. Coronavirus Disease (COVID-19) Pandemic. Available at: https://www.who.int/emergencies/diseases/novel-coronavirus-2019 [Cited on $3^{\text {rd }}$ June 2020 at 05:34 $\mathrm{pm}]$.

3. Van Doremalen N, Bushmaker T, Morris DH, Holbrook MG, Gamble A, Williamson BN, et al. Aerosol and Surface Stability of SARS-CoV-2 as Compared with SARS-CoV-1. N Engl J Med. 2020;382(16):1564-7.

4. Ganguly P, Yunus M, Khan A, Malik A. A study of nosocomial infection about different host factors in an Indian teaching hospital. J R Soc Health. 1995;115(4):244-6.

5. American Dental Association. ADA Calls Upon Dentists to Postpone Elective Procedures. Available at: https://www.ada. org/en/press-room/news-releases/2020-archives/march/adacalls-upon-dentists-to-postpone-elective-procedures [Cited on $3^{\text {rd }}$ June 2020 at 05:40 pm].

6. Ren YF, Rasubala L, Malmstrom H, Eliav E. Dental Care and Oral Health under the Clouds of COVID-19 [published online ahead of print, 2020 Apr 24]. JDR Clin Trans Res. 2020;2380084420924385. doi:10.1177/2380084420924385.

7. Dental Council of India. Precautionary and preventive measures to prevent spreading of Novel Coronavirus (COVID- 19). Available at: http://dciindia.gov.in/Admin/NewsArchives/L. No._8855..PDF [Cited on $3^{\text {rd }}$ June 2020 at 05:48 pm].

8. Harrel SK, Molinari J. Aerosols and splatter in dentistry: A brief review of the literature and infection control implications. J Am Dent Assoc. 2004;135(4):429-37.

9. Alharbi A, Alharbi S, Alqaidi S. Guidelines for dental care provision during the COVID-19 pandemic. Saudi Dent J. 2020;32(4):181-6.

10. Deolia S. Knowledge, Attitude, and Perceived Confidence in the Management of Medical Emergencies in the Dental Office : A Survey among the Nurses in Dental Colleges. DMIMS J Dent Res. 2018;2(2):48-53. 
11. Parveen S, Jain S. Pathophysiologic Enigma of COVID-19 Pandemic With Clinical Correlates. Int J Curr Res Rev. 2020;12(13):33-37

12. Ministry of Health and Family Welfare. Directorate General of Health Services (Emergency Medical Relief). Standard Operating Procedure (SOP) for transporting a suspect/confirmed case of COVID-19. Available at: https://www.mohfw.gov.in/pdf/ StandardOperatingProcedureSOPfortransportingasuspectorconfirmedcaseofCOVID19.pdf [Cited on $3^{\text {rd }}$ June 2020 at 05:55 $\mathrm{pm}]$.

13. Ministry of Health and Family Welfare. Directorate General of Health Services (Emergency Medical Relief). COVID-19 PreScreening Form. Available at: https://dphhs.mt.gov/Portals/85/ publichealth/documents/EMSTS/COVID/COVID19ScreeningForm.pdf[Cited on $3^{\text {rd }}$ June 2020 at 05:59 pm].

14. Ather A, Patel B, Ruparel NB, Diogenes A, Hargreaves KM. Coronavirus Disease 19 (COVID-19): Implications for Clinical Dental Care. J Endod. 2020;46(5):584-595.

15. Ministry of Health and Indigenous Medical Services (Epidemiology Unit). World Health Organization. Guidelines for Home Quarantine. Available on: https://www.who.int/docs/ default-source/searo/whe/coronavirus 19/the-guideline-forhome-quarantine---quarantine-in-non-health-care-settings-isintended-for-anyone-who-believes-they-have-been-exposedto-COVID-19-and-are-required-to-be-home-quarantined-toprevent-community-trans.pdf?sfvrsn $=1 \mathrm{bc} 12565{ }^{4}$ [Cited on $3^{\text {rd }}$ June 2020 at $06: 10 \mathrm{pm}]$.

16. More SM, Kharwade RS, A coordinated roadmap to grip pandemic COVID-19. Coronaviruses. 2020; 1:1-15. DOI: 10.2174/ 2666796701999200801023110.

17. Sen S. Perceptions of Dental Outpatients Towards Cross-Infection Control Measures. DMIMS J Dent Res. 2017;1(4):134-40.

18. Peng X, Xu X, Li Y, Cheng L, Zhou X, Ren B. Transmission routes of 2019-nCoV and controls in dental practice. Int J Oral Sci. 2020;12(1):9.

19. Kariwa H, Fujii N, Takashima I. Inactivation of SARS coronavirus employing povidone-iodine, physical conditions, and chemical reagents. Jpn J Vet Res. 2004;52(3):105-12.

20. Centres for Disease Control and Prevention. Sequence for Putting on and Removing Personal Protective Equipment. Available on: https://www.cdc.gov/hai/pdfs/ppe/PPE-Sequence.pdf [Cited on 3rd June 2020 at $06.20 \mathrm{pm}$ ].

21. Ministry of Health and Family Welfare Directorate General of Health Services (Emergency Medical Relief). Coronavirus Disease 2019 (COVID-19): Guidelines on the rational use of Personal Protective Equipment. Available at: https://www.mohfw. gov.in/pdf/GuidelinesonrationaluseofPersonalProtectiveEquipment.pdf [Cited on 3rd June 2020 at 06.24 pm].

22. Centres for Disease Control and Prevention. Interim Infection Prevention and Control Recommendations for Patients with Suspected or Confirmed Coronavirus Disease 2019 (COVID-19) in Healthcare Settings. Available at: https://www.cdc. gov/coronavirus/2019-ncov/hcp/infection-control-recommendations.html\# [Cited on 3rd June 2020 at 06.50 pm].

23. United States Environmental Protection Agency. List N: Disinfectants for Use Against SARS-CoV-2. Available at: https:// www.epa.gov/pesticide-registration/list-n-disinfectants-useagainst-sars-cov-2 [Cited on 3rd June 2020 at $06.58 \mathrm{pm}$ ].

24. Chin A, Chu J, Perera M, Hui K, Yen H-L, Chan M, et al. Stability of SARS-CoV-2 in different environmental conditions. The Lancet Microbe. 2020;1(1):e10.

25. Kampf G, Todt D, Pfaender S, Steinmann E. Persistence of coronaviruses on inanimate surfaces and their inactivation with biocidal agents. J Hosp Infect. 2020;104(3):246-51.

26. Nimonkar SV, Belkhode VM, Godbole SR, Nimonkar PV, Dahane T, Sathe S. Comparative Evaluation of the Effect of Chemical Disinfectants and Ultraviolet Disinfection on Dimensional Stability of the Polyvinyl Siloxane Impressions. J IntSocPrev Community Dent. 2019;9(2):152-8.

27. Biradar S. Sterilization in Dental Practice - A Review. Asian J Pharm TechnolInnov. 2018;6(27):53-66.

28. Ramasamy K,Jayakumar S. The Trend of COVID-19 at Bengaluru: Prediction to Continue the Better Epidemic Management. International Journal of Current Research and Review. 2020;12(13):56-60. 10.31782/IJCRR.2020.121310.

29. Ramasamy K,Jayakumar S, Chinnu G. COVID-19 Situation at Chennai City -Forecasting for the Better PandemicManagement. International Journal of Current Research and Review. 2020;12(12):37-47. 